\title{
QUEEN'S
UNIVERSITY
BELFAST
}

\section{Changes in modal parameters of a steel truss bridge due to artificial damage}

Kim, C. W., Chang, K. C., Kitauchi, S., McGetrick, P. J., Hashimoto, K., \& Sugiura, K. (2014). Changes in modal parameters of a steel truss bridge due to artificial damage. In G. Deodatis, B. R. Ellingwood, \& D. M. Frangopol (Eds.), Safety, Reliability, Risk and Life-Cycle Performance of Structures and Infrastructures - Proceedings of the 11th International Conference on Structural Safety and Reliability, ICOSSAR 2013 (pp. 3725-3732). CRC Press. https://www.crcpress.com/Safety-Reliability-Risk-and-Life-Cycle-Performance-of-Structuresand/Deodatis-Ellingwood-Frangopol/9781138000865

Published in:

Safety, Reliability, Risk and Life-Cycle Performance of Structures and Infrastructures - Proceedings of the 11th International Conference on Structural Safety and Reliability, ICOSSAR 2013

\section{Document Version:}

Peer reviewed version

Queen's University Belfast - Research Portal:

Link to publication record in Queen's University Belfast Research Portal

\section{Publisher rights}

(c) 2014 CRC/Balkema

Published in Safety, Reliability, Risk and Life-Cycle Performance of Structures and Infrastructures

\section{General rights}

Copyright for the publications made accessible via the Queen's University Belfast Research Portal is retained by the author(s) and / or other copyright owners and it is a condition of accessing these publications that users recognise and abide by the legal requirements associated with these rights.

Take down policy

The Research Portal is Queen's institutional repository that provides access to Queen's research output. Every effort has been made to ensure that content in the Research Portal does not infringe any person's rights, or applicable UK laws. If you discover content in the Research Portal that you believe breaches copyright or violates any law, please contact openaccess@qub.ac.uk. 


\title{
Changes in Modal Parameters of a Steel Truss Bridge due to Artificial Damage
}

\author{
C.W. Kim, K.C. Chang, S. Kitauchi, P.J. McGetrick, K. Hashimoto \& K. Sugiura \\ Dept. of Civil and Earth Resources Eng., Graduate School of Eng., Kyoto University, Kyoto 615-8540, Japan
}

\begin{abstract}
This study investigated how damage changes the modal parameters of a real bridge by means of a field experiment which was conducted on a real steel truss bridge consecutively subjected to four artificial damage scenarios. In the experiment, both the forced and free vibrations of the bridge were recorded, the former for identifying higher modes available exclusively and the latter for lower modes with higher resolution. Results show that modal parameters are little affected by damage causing low stress redistribution. Modal frequencies decrease as damage causing high stress redistribution is applied; such a change can be observed if the damage is at the non-nodal point of the corresponding mode shape. Mode shapes are distorted due to asymmetric damage; they show an amplification in the damaged side as damage is applied at the non-nodal point. Torsion modes become more dominant as damage is applied either asymmetrically or on an element against large design loads.
\end{abstract}

\section{INTRODUCTION}

Over last few decades, bridge health monitoring (BHM) has drawn increasing attention, especially in those developed countries or areas where a considerable amount of bridges were constructed in rapid economic development periods and hence are approaching their design lives. A key task in BHM is to detect, localize or quantify possible damage via any effective technique. One popular nondestructive detection technique is based on bridge vibrations (referred to as vibration-based damage detection, VBDD, hereafter), with the intuitive basis that damage in a bridge will change the mechanical properties of the bridge, such as stiffness, mass or damping, and hence change the bridge's dynamic responses in the time domain as well as dynamic characteristics, such as modal parameters or their derivatives, in the frequency or modal domain (Farrar et al. 2001, Carden \& Fanning 2004, Fan \& Qiao 2011).

To examine practicability of various VBDD techniques on 'damaged bridges,' damage is required to introduce into test bridges. Damage can be more easily implemented, say with less cost in money and effort, in numerical bridge models and scaled laboratory bridge models than in full-scale real bridges. Moreover, damage is generally not allowed to apply on existing in-service bridges. Partly due to the above reasons, relevant field experimental studies are still limited in number, in comparison with nu- merical studies and laboratory experimental studies. To authors' knowledge, there were several field damage experiments conducted in recent two decades for testing VBDD techniques on the following bridges: I-40 Bridge, a steel plate girder bridge (Farrar et al. 1994); Z24 Bridge, a pre-stressed concrete (PC) box girder bridge (Kramer et al. 1999); Romeo Bridge, a PC box girder bridge (Huth at al. 2005); Kurumakaeri Bridge, a steel truss bridge (Furukawa \& Otsuka 2008); Dogna Bridge, a reinforced concrete (RC) girder bridge (Dilena \& Morassi 2011); Torigai Bridge, a steel truss bridge (Kim et al. 2013); a steel truss bridge (no name given) (Yoshioka et al. 2011); Deutsche Bank Bridge, a PC slab bridge (Maas et al. 2012); and Avenue John F. Kennedy Bridge, a PC box girder bridge (Maas et al. 2012). Although many valuable results have been yielded, the number of field experiments is still insufficient to guarantee the practicability and reliability of existing VBDD techniques on real bridges. For practical VBDD, more understanding of how damage changes the dynamic characteristics of a real bridge is always highly expected.

This study presents the results of a field damage experiment conducted on a real simply-supported steel truss bridge and investigates how the modal parameters of the bridge change due to artificial damage. The bridge was consecutively subjected to the following artificial damage: 1) a half cut in the vertical member at midspan, 2) a full cut in the same member, 3 ) recovery of the full cut, and 4) a full cut 
in vertical member at $5 / 8$ th span. In the experiment, the bridge was excited by the passage of a two-axle commercial van, and both the bridge forcedvibration $(\mathrm{FoV})$ responses, excited during the vehicle passage, and free-vibration (FrV) responses, excited after the vehicle passage, are recorded for identifying modal parameters. The modal parameters, including the modal frequency and mode shape, are identified with the frequency domain decomposition (FDD). Focus is put on the change of those modal parameters to the artificial damage and on the mechanical interpretations of the changes. Besides, the results obtained from forced and free vibration responses are compared statistically, to investigate how those results complement each other and whether the passing vehicle may affect the modal parameters or not.

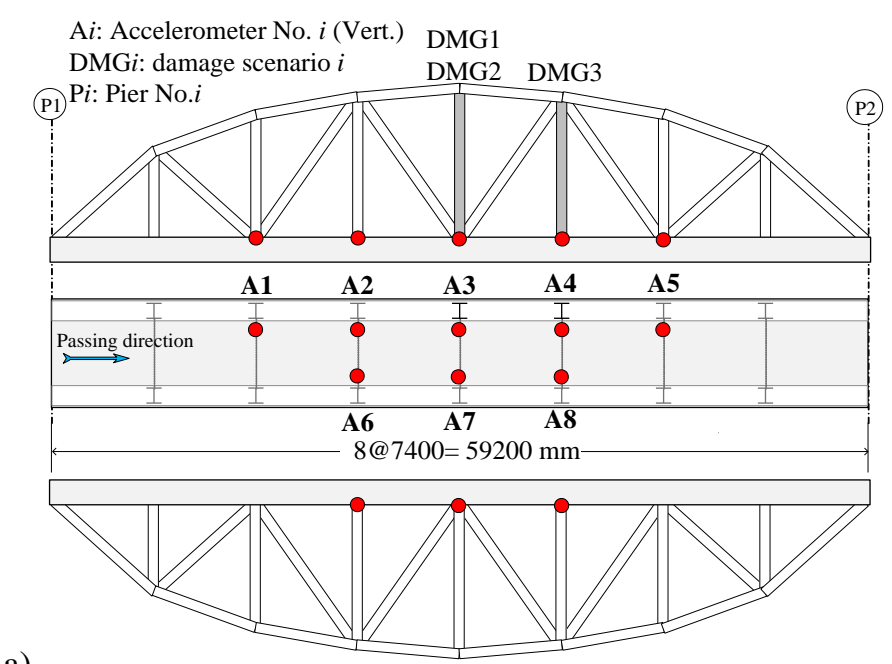

a)

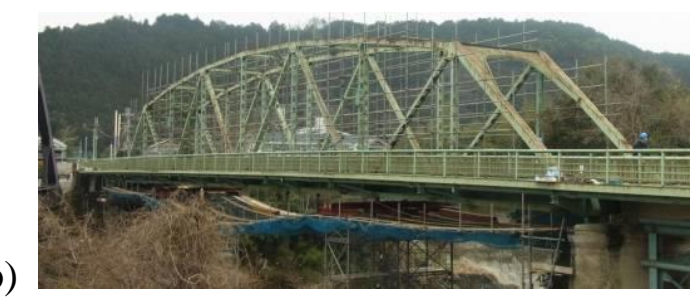

Figure 1. Experiment bridge: a) sketch along with sensor layout; b) elevation photo.

\section{EXPERIMENT DESCRIPTIONS}

\subsection{Experiment bridge and vehicle}

The experiment bridge was a simply-supported through-type steel Warren truss bridge, as shown in Figure 1. It was $59.2 \mathrm{~m}$ in span length, $8.2 \mathrm{~m}$ in maximum height, and $3.6 \mathrm{~m}$ in width, designed for a single lane. The bridge was constructed in 1959 and planned to remove after a new replacement bridge was open to traffic in 2012. Before its removal, the bridge was allowed an artificial damage experiment. During the experiment, the bridge had been closed to traffic, and therefore no other vehicles besides the experiment vehicle were allowed.

The experiment vehicle was a two-axle commercial van, Serena model produced by Nissan Motor Company Ltd. The total weight was about $21 \mathrm{kN}$. No dynamic pre-test was performed on the van so that no prior van's dynamic property is known.

Table 1. Damage scenario.

\begin{tabular}{ll}
\hline Scenario & Description \\
\hline INT & Intact bridge \\
DMG1 & Half cut in vertical member at midspan \\
DMG2 & Full cut in vertical member at midspan \\
RCV & Recovery of the cut member \\
DMG3 & Full cut in vertical member at 5/8th span \\
\hline
\end{tabular}

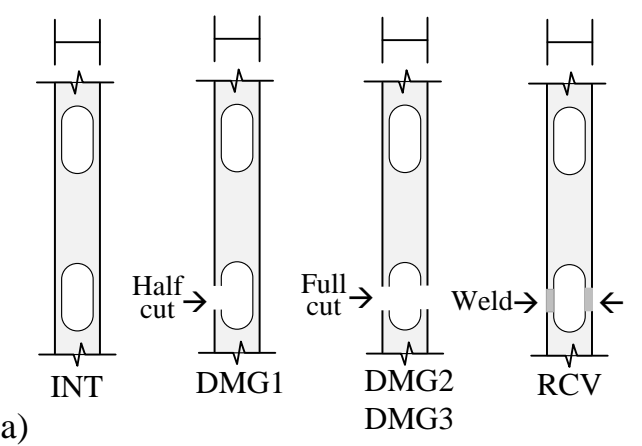

a)
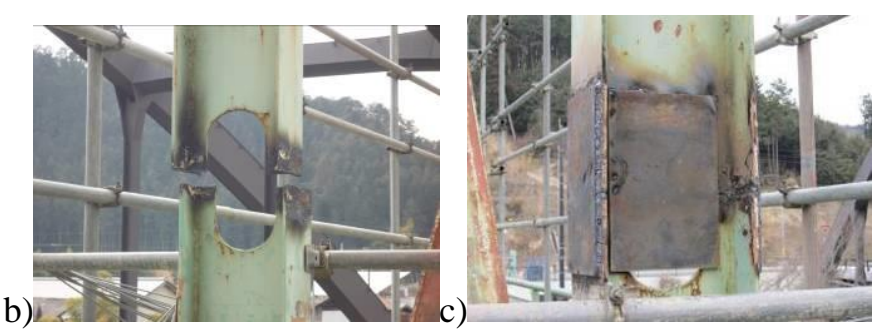

Figure 2. Artificial damage: a) sketch; b) photo of the full cut; c) photo of the recovery of the full cut.

\subsection{Damage scenarios}

Five scenarios are considered in this study, as briefly summarized in Table 1 and sketched in Figure 2a). Initially, the INT scenario denotes the intact bridge with no artificial damage. Then, two damage scenarios were applied consecutively. The first damage (DMG1) denotes a half cut applied by a cutting torch in the vertical member at the mid-span (see Fig. 1a); the second damage (DMG2) denotes a full cut (see Fig. 2b) applied in the same member. Those applied cuts were designed to imitate real damage patterns that had been inspected previously, probably caused by corrosion or overloading. After DMG2, the full cut was recovered (see Fig. 2c), which is denoted as the RCV scenario. Although the recovery was made by utilizing a jack to reduce the gap in the cut member before welding steel plates on it, the bridge was not guaranteed to restore to its original intact state. It does not matter whether the bridge was restored to its original state or not; the RCV scenario simply 
served as a reference to the following damage scenario, DMG3 scenario: a full cut applied in the vertical member located at the 5/8th span (see Fig. 1a).

The three damage scenarios, DMG1, DMG2, and DMG3 scenarios, represent different typical damage mechanisms, respectively. DMG1 scenario represents a damage mechanism with low stress redistribution; DMG2 scenario represents a damage mechanism with high stress redistribution; and DMG3 scenario represents another damage mechanism with high stress redistribution, but different from DMG2 scenario in a) symmetry: DMG2 is symmetric damage because it was applied at the midspan and hence supposed to yield a symmetric stress redistribution, while DMG3 is asymmetric damage because it was applied at the non-midspan and hence supposed to yield an asymmetric stress re-distribution; and b) design axial force $\left(F_{d}\right)$ against dead loads: $F_{d}$ is 33.717 $\mathrm{kN}(3.437 \mathrm{tf})$ for DMG2 element and $101.926 \mathrm{kN}$ (10.390 tf), about three times the former, for DMG3 element. In addition, DMG3 element is the vertical member with largest $F_{d}$.

\subsection{Test types and layouts}

As shown in Figure 1, eight uni-axial accelerometers were installed vertically on the experiment bridge, five at the damage side (A1-A5) and three at the opposite side (A6-A8). Limited to the number of accelerometers available, the A6-A8 side serves to offer a clue to the judgment of torsion modes, rather than to construct complete mode shapes. Two optical sensors were installed at two ends of the bridge, respectively, to detect the entrance and exit of the vehicle. The sampling frequency was set as $200 \mathrm{~Hz}$ for all sensors.

A moving vehicle experiment was performed on the experiment bridge. During the experiment, the bridge was travelled by the experiment vehicle, and the bridge vertical acceleration responses, both during and after the vehicle passage, were measured by the accelerometers. Ten runs were conducted for all scenarios except DMG1 scenario, for which twelve runs were conducted.

Although the vehicle was planned to move at a constant speed, it is difficult, even impossible, to maintain the constant speed manually. Under this difficulty, the travelling speed was calculated in an average way by dividing the known bridge span length by the travelling time length measured by the optical sensors. The average speed such calculated ranged from 36 to $41 \mathrm{~km} / \mathrm{hr}$.

The temperature was not recorded, but no dramatic temperature variation was felt during the experiment, which was performed in the daytime of two consecutive days in late winter. Therefore, the effect of the temperature on the bridge can be reasonably neglected.

\section{MODAL IDENTIFICATION METHOD}

The recorded acceleration responses are performed with the frequency domain decomposition (FDD), a frequency-domain analysis technique in identifying structural modal parameters from output responses only. It is popular since it is fast, simple, and userfriendly to operate, and offers users intuitively physical understanding on the data under analysis. Its algorithm can be briefly described as follows.

Suppose that $M$ sets of time-history responses of the bridge were measured. First, estimate the $M$-by$M$ power spectrum density (PSD) matrix $S\left(i \omega_{j}\right)$, where $\omega_{j}$ denotes $j$-th discrete frequency. Then, for every discrete frequency, decompose $S\left(i \omega_{j}\right)$ by the singular value decomposition (SVD):

$$
S\left(i \omega_{j}\right)=U_{j} \Sigma_{j} U_{j}^{H}
$$

where $\boldsymbol{U}_{j}=\left[u_{j l}, u_{j 2}, \ldots, u_{j M}\right]$ is an unitary matrix composed of the singular vectors $u_{j k}, \sum_{j}$ a diagonal matrix composed of $M$ scalar singular values $s_{j k}$, and $H$ denotes a conjugate transpose operation. Plotting singular values with respect to frequency to yield a singular value spectrum (SV spectrum hereinafter), one may identify a structural modal frequency, e.g. $\omega_{b}$, by picking the frequency value where a peak singular value appears. The corresponding mode shape can be obtained as the first singular vector $u_{b 1}$ corresponding to the modal frequency $\omega_{b}$.

The algorithm described above is of the simplest version - the peak-picking version - of FDD. More advanced versions and their related theoretical backgrounds are available in other literature (Brincker et al. 2000, 2001).

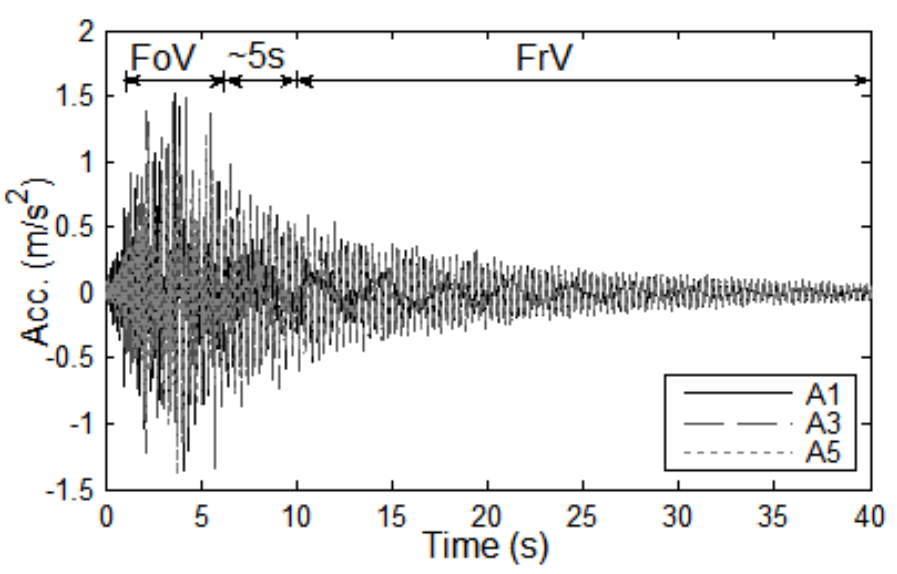

Figure 3. Time history of bridge acceleration responses.

\section{RESULTS \& DISCUSSIONS}

Let us take a typical case, e.g. Run 1 of the INT scenario, for example. Figure 3 shows the time history of the bridge acceleration responses recorded from accelerometers A1, A3 and A5. In the time history, the FoV interval is labeled for about the first 1-6 
seconds, and the FrV interval for a 30-second span about 5 seconds behind the FoV interval. A 5-second spacing between FrV and FoV intervals was adopted for securing the bridge responses fully free from the vehicle-loading effect. In FoV interval, larger acceleration amplitudes induced by the vehicle passage can be observed; in FrV interval, a clear amplitudedecay is observed.

Performing FDD on the FoV and FrV acceleration responses recorded from all eight accelerometers, the spectra of the largest SV spectrum can be obtained, as plotted in Figures 4a) and b), respectively. The modal frequencies $f_{i}$ 's are identified by picking the peaks appearing in those SV spectra, and the corresponding mode shapes $\varphi_{i}$ 's are exactly the first singular vectors calculated at $f_{i}$ 's.
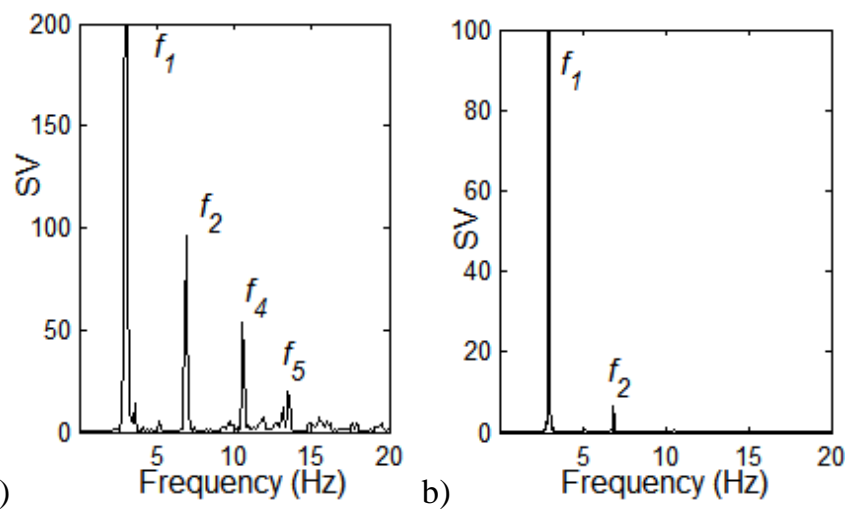

Figure 4. Singular value spectra: a) forced vibration response; b) free vibration response.

\subsection{Identified modal parameters}

With the same algorithm, the first several modal frequencies and mode shapes can be identified for every all runs of all scenarios. For the first five modes, the identified modal frequencies and the mean mode shapes with respect to all scenarios are plotted in Figure 5. Their statistical results, including the numbers (No) of run with the modal frequency successfully identified, means (M), standard deviations (STD), and coefficients of variance (CV), are summarized in Table 2. Note that the mode shapes given in Figure 5 are from FrV for the first three modes and from FoV for the fourth and fifth modes, with reasons given in Section 4.2; the FrV results for the fourth and fifth modes are not given in Table 3, because in too few runs (mostly no more than two) can the modal frequency be successfully identified. The five identified modes should correspond to bending modes because the mode shapes of the two sides (the A1-A5 and A6-A8 sides) are in phases (see Figure 5, where the A1-A5 side is represented by the longer curve and A6-A8 side by the shorter curve).

The accuracy and precision of the identified modal frequencies are high. The accuracy can be verified from both FrV and FoV responses mutually. Taking $f_{1}$ of INT scenario for example, it is identified as
$2.980 \mathrm{~Hz}$ in mean from $\mathrm{FrV}$ responses and is very close to that, $2.964 \mathrm{~Hz}$, identified from FoV responses (see Table 2). The close identified frequencies from both responses can be observed in other modal frequencies as well as other scenarios. On the other hand, the precision of the identified modal frequencies is high because the CVs of the modal frequencies are small, generally smaller than $1 \%$.
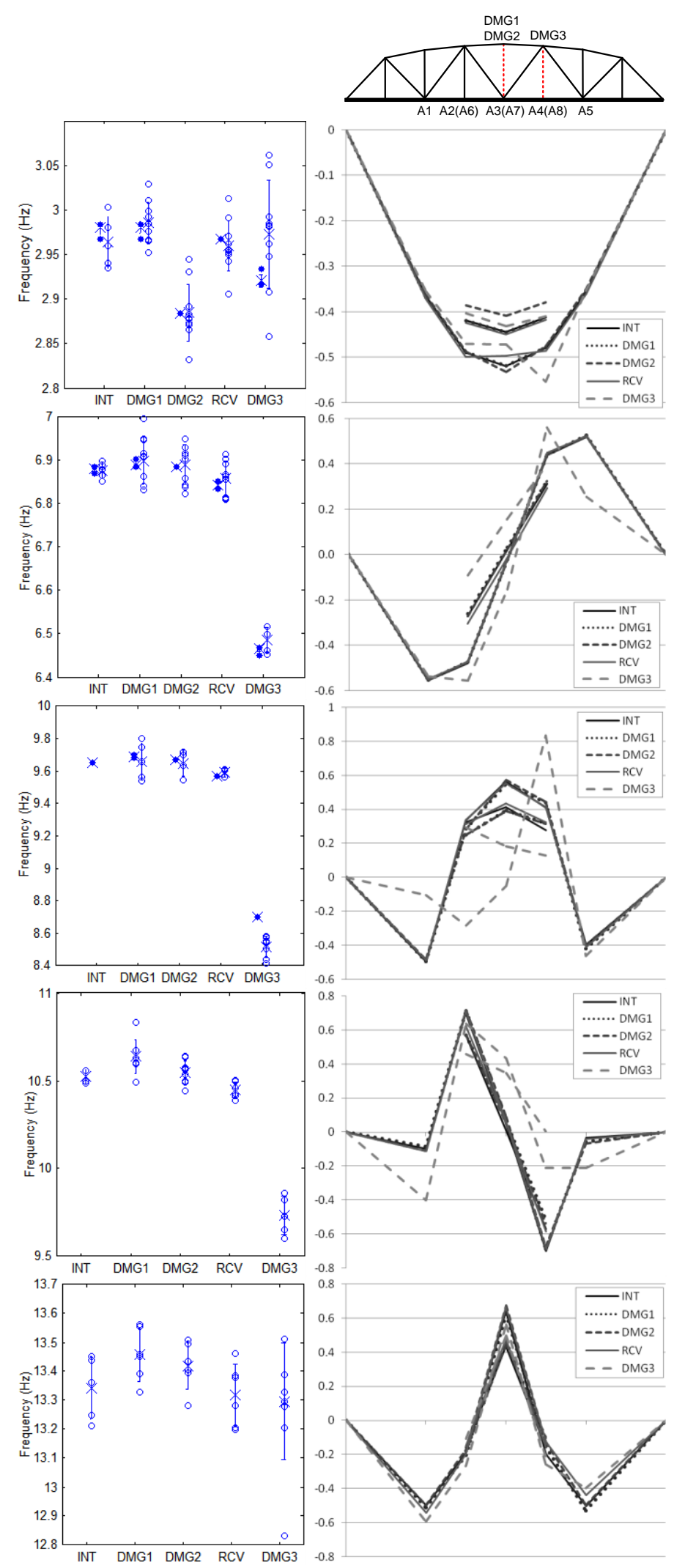

Figure 5. Identified modal frequencies (left) and mean mode shapes (right) of the first five modes (from top to bottom). ( $\bullet$ : FrV, o: FoV, x: mean, error bar: $\pm \sigma$ ) 
Table 2. Statistical properties of the first five modal frequencies identified from FrV and FoV responses.

\begin{tabular}{|c|c|c|c|c|c|c|c|}
\hline & & & INT & DMG1 & DMG2 & $\mathrm{RCV}$ & DMG3 \\
\hline \multicolumn{8}{|c|}{$\overline{f_{1}}$} \\
\hline & \multirow[t]{4}{*}{ FrV } & No & $10 / 10$ & $12 / 12$ & $10 / 10$ & $10 / 10$ & $10 / 10$ \\
\hline & & $\mathrm{M}$ & 2.980 & 2.981 & 2.884 & 2.967 & 2.920 \\
\hline & & STD & 0.007 & 0.006 & 0.000 & 0.000 & 0.007 \\
\hline & & $\mathrm{CV}$ & $0.22 \%$ & $0.22 \%$ & $0.00 \%$ & $0.00 \%$ & $0.24 \%$ \\
\hline & \multirow[t]{4}{*}{ FoV } & No & $5 / 5$ & $12 / 12$ & $10 / 10$ & $10 / 10$ & $10 / 10$ \\
\hline & & $\mathrm{M}$ & 2.964 & 2.986 & 2.885 & 2.960 & 2.973 \\
\hline & & STD & 0.028 & 0.022 & 0.032 & 0.029 & 0.060 \\
\hline & & $\mathrm{CV}$ & $0.96 \%$ & $0.74 \%$ & $1.11 \%$ & $0.97 \%$ & $2.03 \%$ \\
\hline \multicolumn{8}{|c|}{$f_{2}$} \\
\hline & \multirow[t]{4}{*}{ FrV } & No & $9 / 10$ & $10 / 12$ & $7 / 10$ & $4 / 10$ & $8 / 10$ \\
\hline & & M & 6.878 & 6.889 & 6.884 & 6.842 & 6.465 \\
\hline & & STD & 0.008 & 0.008 & 0.000 & 0.010 & 0.006 \\
\hline & & $\mathrm{CV}$ & $0.12 \%$ & $0.12 \%$ & $0.00 \%$ & $0.14 \%$ & $0.09 \%$ \\
\hline & \multirow[t]{4}{*}{ FoV } & No & $5 / 5$ & $12 / 12$ & $10 / 10$ & $10 / 10$ & $5 / 10$ \\
\hline & & $\mathrm{M}$ & 6.875 & 6.898 & 6.889 & 6.858 & 6.485 \\
\hline & & STD & 0.019 & 0.055 & 0.045 & 0.037 & 0.028 \\
\hline & & $\mathrm{CV}$ & $0.27 \%$ & $0.79 \%$ & $0.65 \%$ & $0.54 \%$ & $0.43 \%$ \\
\hline \multirow[t]{9}{*}{$\overline{f_{3}}$} & \multirow{5}{*}{ FrV } & & & & & & \\
\hline & & No & $1 / 10$ & $6 / 12$ & $7 / 10$ & $1 / 10$ & $1 / 10$ \\
\hline & & M & 9.651 & 9.687 & 9.667 & 9.567 & 8.701 \\
\hline & & STD & $x$ & 0.007 & 0.000 & $x$ & $x$ \\
\hline & & $\mathrm{CV}$ & $x$ & $0.07 \%$ & $0.00 \%$ & $x$ & $x$ \\
\hline & \multirow[t]{4}{*}{ FoV } & No & $0 / 5$ & $5 / 12$ & $4 / 10$ & $3 / 10$ & $7 / 10$ \\
\hline & & M & $x$ & 9.661 & 9.648 & 9.591 & 8.516 \\
\hline & & STD & $x$ & 0.113 & 0.079 & 0.027 & 0.066 \\
\hline & & $\mathrm{CV}$ & $x$ & $1.17 \%$ & $0.82 \%$ & $0.28 \%$ & $0.78 \%$ \\
\hline \multicolumn{8}{|c|}{$\overline{f_{4}}$} \\
\hline & \multirow[t]{4}{*}{ FoV } & No & $4 / 5$ & $8 / 12$ & $9 / 10$ & $7 / 10$ & $5 / 10$ \\
\hline & & M & 10.525 & 10.638 & 10.548 & 10.449 & 9.731 \\
\hline & & STD & 0.038 & 0.097 & 0.065 & 0.043 & 0.110 \\
\hline & & $\mathrm{CV}$ & $0.36 \%$ & $0.91 \%$ & $0.62 \%$ & $0.42 \%$ & $1.13 \%$ \\
\hline \multirow[t]{5}{*}{$\overline{f_{5}}$} & & & & & & & \\
\hline & \multirow[t]{4}{*}{ FoV } & No & $5 / 5$ & $6 / 12$ & $6 / 10$ & $6 / 10$ & $9 / 10$ \\
\hline & & $\mathrm{M}$ & 13.343 & 13.457 & 13.420 & 13.318 & 13.297 \\
\hline & & STD & 0.109 & 0.091 & 0.082 & 0.107 & 0.202 \\
\hline & & $\mathrm{CV}$ & $0.81 \%$ & $0.68 \%$ & $0.61 \%$ & $0.80 \%$ & $1.52 \%$ \\
\hline
\end{tabular}

No: number of runs with the modal frequency successfully identified / total number of runs; M: mean [Hz]; STD: standard deviation $[\mathrm{Hz}]$; $\mathrm{CV}$ : coefficient of variance; $\mathrm{x}$ : unavailable.

Suggested by the close identified mean frequencies from FrV and FoV responses, the moving experiment vehicle hardly induces variation in bridge modal frequencies via vehicle-bridge interactions (VBI), probably because it is too light in compared with the experiment bridge (Chang et al. 2013). More specifically speaking, the vehicle and bridge may interact with each other and form a VBI system whose vibrating modes may vary from those of the bridge system alone. In theory, the vibrating modes extracted from FoV responses should relate to the VBI system, while those from FrV responses to the bridge system alone. In this experiment, the modal frequency identified from those two types of response show little difference, indicating that the vibrating modes of the bridge in VBI system closely approximate to and thus can be reasonably regarded as those of the bridge alone.

\subsection{Forced vs. free vibration responses}

The difference between results identified from FoV and FrV responses can be observed from Figure 5 and Table 2, as well as a graphical presentation of Table 2 in Figures 6 and 7, where the former shows the number (No) of run with the modal frequency successfully identified and the latter shows the coefficient of variance $(\mathrm{CV})$.

Firstly, the FoV responses offer more information of higher modes than FrV responses do, as can be seen in Figure 4, where more peaks appear in the FoV SV spectra, and in Figure 6, where generally in a greater or equal number of FoV runs can the modal frequencies be successfully identified, especially for higher modes. The FoV responses can offer more information majorly because they are excited by more energy input from vehicle loadings.

Secondly, the FrV responses offer more precise results than FoV responses do, as can be observed in Table 2 and Figure 7, where the CVs of FrV responses are smaller than those of FoV responses for all modes and all scenarios. It may be resulted from the following factors. One is the freedom of FrV responses from vehicle loadings and thus from a possible, although slight, variation in the bridge modal parameters caused by VBIs. Another one is the longer time interval that can be taken for analysis, offering a higher resolution in SV spectra. Note that the second factor may not be true once the experiment bridge is subjected to a large damping effect.

Based on the above observations, it is suggested to interpret the identified modal parameters of lower modes from FrV responses, for higher resolution and precision purposes, and those of higher modes that are available exclusively from FoV responses. In this study, the first three modes from FrV responses and those of the fourth and fifth modes from FoV responses are taken to interpret, as done in Section 4.1 and the following section.

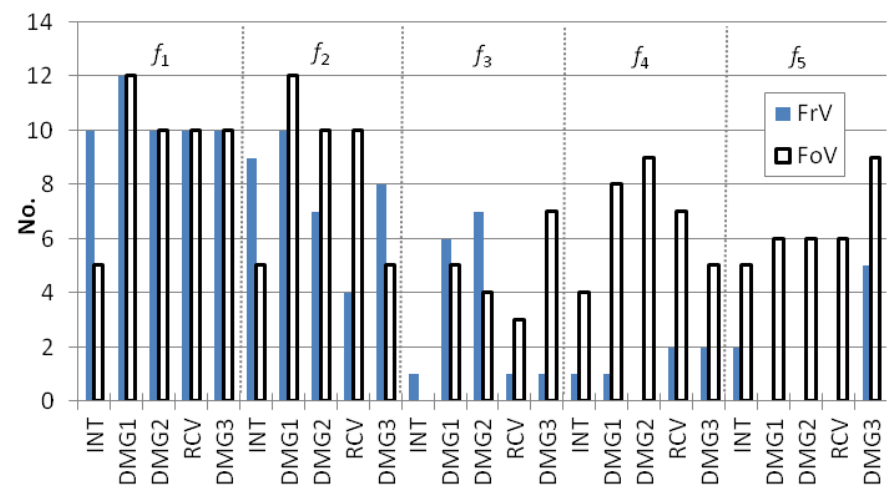

Figure 6. Number of runs with the modal frequency successfully identified. 


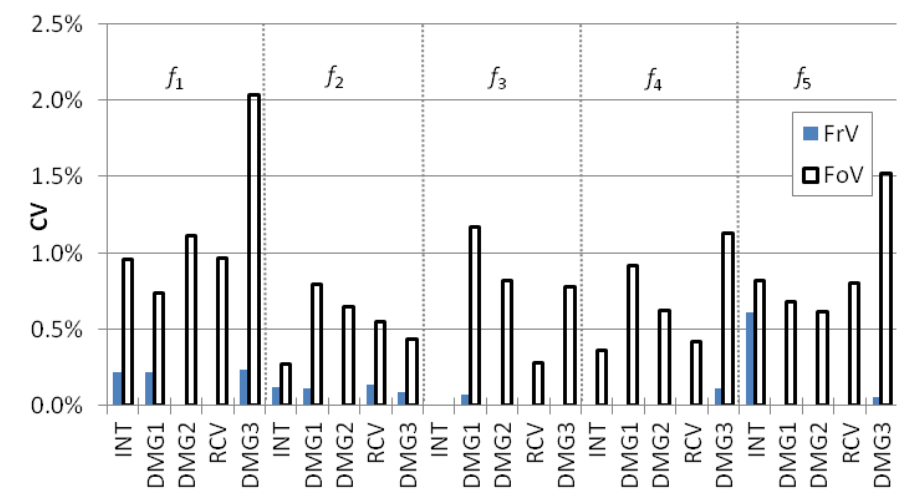

Figure 7. Coefficient of variance.

Table 3. Change of modal frequency $f_{i}$ and mode shape $\varphi_{i}$ due to different damage.

\begin{tabular}{lll}
\hline Scenario & $f_{i}$ & $\varphi_{i}$ \\
\hline 1st bending mode* & & \\
DMG1 & $+0.03 \%$ & Little variation \\
DMG2 & $-3.22 \%$ & Conspicuous in damaged side \\
DMG3 & $-1.58 \%$ & Distortion \\
2nd bending mode* & & \\
DMG1 & $+0.16 \%$ & Little variation \\
DMG2 & $+0.09 \%$ & Little variation \\
DMG3 & $-5.51 \%$ & Distortion \\
3rd bending mode* & & \\
DMG1 & $\times$ & $\times$ \\
DMG2 & $-0.21 \%$ & Conspicuous in damaged side \\
DMG3 & $-9.05 \%$ & Distortion; mode mixture \\
4th bending mode $* *$ & & \\
DMG1 & $+1.07 \%$ & Little variation \\
DMG2 & $+0.22 \%$ & Little variation \\
DMG3 & $-6.87 \%$ & Distortion \\
5th bending mode** & & \\
DMG1 & $+0.85 \%$ & Little variation \\
DMG2 & $+0.58 \%$ & Little variation \\
DMG3 & $-0.16 \%$ & Slight distortion \\
1st torsion mode* & & \\
DMG1 & $\times$ & $\times$ \\
DMG2 & $\times$ & $\times$ \\
DMG3 & Identified & Identified \\
\hline *: from FrV, **: from FoV. & \\
& & \\
\hline
\end{tabular}

\subsection{Changes in modal parameters}

The changes in modal parameters with respect to each damage scenario can be observed from Figure 5 and summarized in Table 3. Note that the changes are based on INT scenario for DMG1 or DMG2 scenario and on RCV scenario for DMG3 scenario; that the changes in modal frequencies are expressed quantitatively in percentage and those in mode shapes are inspected qualitatively by appearance.

For the 1st bending mode, both the modal frequency $f_{1}$ and modal shape $\varphi_{1}$ show little change as DMG1 is applied, indicating that damage causing low stress redistribution may have little effect on the modal parameters. As DMG2 is applied, $f_{1}$ decreases up to $3 \%$, and $\varphi_{1}$ shows an observable amplification in the damaged side (equivalent to a reduction in the opposite side, as shown in Figure 5), indicating that damage causing high stress redistribution may reduce the global bridge stiffness and therefore result in an observable change in the modal parameters. Furthermore, little distortion is observed in $\varphi_{1}$, probably because that the symmetric, although severe, damage may hardly distort the symmetric mode shape. As DMG3 is applied, $f_{1}$ decreases about $1.6 \%$, indicating an observable loss in the global stiffness; $\varphi_{1}$ is distorted, illustrating that asymmetric damage may distort the symmetric mode shape. Moreover, the local amplification in mode shape, or mechanically the local loss in stiffness, due to damage is also observed, e.g. see A3 point in DMG2 scenario and A4 in DMG3.

For the 2nd bending mode, both the modal frequency $f_{2}$ and mode shape $\varphi_{2}$ show little change as DMG1 is applied, like those for the 1st bending mode do. However, unlike the 1 st bending mode, $f_{2}$ and $\varphi_{2}$ show little change as DMG2 is applied. Ostensibly such an observation seems to violate one's mechanical sense: severe damage should cause a loss in global stiffness and be signified by changes in modal parameters. The above mechanical statement is still true, but little change in the 2 nd bending modal parameters observed herein may suggest a possibility: damage applied at the node of a mode shape does not affect the vibrating modes. It is mechanically reasonable in that elements in the opposite sides of a node, vibrating in antiphase, keep the node in dynamic equilibrium regardless of the magnitude of node stiffness. As DMG3 is applied, $f_{2}$ decreases up to $5.5 \%$, indicating again a large loss in the global stiffness; $\varphi_{2}$ is distorted, illustrating that asymmetric damage may distort an antisymmetric mode shape besides the aforementioned symmetric mode shape.

For the 3rd bending mode, the change of modal parameters from INT to DMG1 scenario is not reliably available because too few run (1 run only) is the modal frequency successfully identified for INT scenario to offer a reliable reference. In contrast, the change of modal parameters from DMG1 to DMG2 scenario is more reliable, each with at least six successful runs. As DMG2 is applied, the 3rd modal frequency $f_{3}$ decreases slightly (about $0.2 \%$ ), and the 3rd mode shape $\varphi_{3}$ shows a slight amplification in the damaged side (reduction in the opposite side) but little shape distortion. It indicates again that, as observed for the 1st mode, damage causing high stress redistribution may reduce the global bridge stiffness and therefore result in an observable change in the modal parameters, but a symmetric damage does not distort the symmetric mode shape. As DMG3 is applied, $f_{3}$ greatly decreases (up to $9 \%$ ), and $\varphi_{3}$ is distorted greatly and perhaps mixed with a certain torsion mode, illustrating again that asymmetric damage may cause a distortion in symmetric mode shapes. However, it should be noted that the changes in modal parameters due to DMG3 is not as reliable as those due to DMG2, because both the reference case, $\mathrm{RCV}$, and the response case, DMG3, are iden- 
tified with too few successful runs (1 run only) to offer high reliability.

For the 4th bending mode, an antisymmetric mode, the changes of the modal frequency $f_{4}$ and mode shape $\varphi_{4}$ follow a similar trend as for the 2 nd bending mode: little change due to both DMG1 and DMG2 but a considerable decrease (up to 6\%) in $f_{4}$ and an obvious distortion in $\varphi_{4}$ due to DGM3. It emphasizes again that damage applied at the node of a mode shape may hardly affect the vibrating modes and that asymmetric damage may not only reduce the global stiffness but also distort an antisymmetric mode shape.

For the 5th bending mode, no obvious change is observed in modal parameters, probably due to too low the vibration level excited by the passing vehicle as well as too sparse the sensor density.

In addition, a mode different from the above bending modes is identified only in DMG3 case but not in other cases. The modal frequency of such mode is identified as $5.10 \mathrm{~Hz}$, and the corresponding mode shape is as shown in Figure 8. This mode can be identified as a torsion mode since the opposite sides are in antiphase with each other. The observation that this mode appears only as DMG3 but not as DMG2 is applied reveals a possibility: either asymmetric damage or damage on the element against large design dead loads may make a torsion mode more dominant in bridge vibrations, probably because, from a mechanical viewpoint, the asymmetric damage breaks the symmetry in global bridge stiffness and makes the torsion mode easier to be excited. Note that the excited torsion mode is the one of the damaged bridge rather than of the original intact bridge.

From the above observations, more general summaries can be given as follows.

- Modal parameters are little affected by damage causing low stress redistribution, such as DMG1.

- Modal frequencies decrease, signifying a global stiffness loss, as damage causing high stress redistribution is applied. Such a change can be observed if the damage is applied at the non-nodal point of corresponding mode shapes, such as the 1 st bending mode in DMG2 and DMG3 scenarios, the 2nd mode in DMG3 scenario, the 3rd bending mode in DMG2 and DMG3 scenarios, and the 4th bending mode in DMG3 scenario. However, the change is not necessarily observable if the damage is applied at the node of the mode shapes, such as the 2nd and 4th bending modes in DMG2 scenario.

- Both symmetric and antisymmetric mode shapes are distorted as asymmetric damage, such as DMG3, is applied. However, the distortion in mode shape is not observed as symmetric damage, such as DMG2, is applied. Alternatively, as damage is applied at the non-nodal point of the mode shapes, such as DMG2 at the 1st and 3rd bending modes, the mode shapes show an amplification in the damaged side (equivalent to a reduction in the opposite side), reflecting a stiffness reduction in this side; as damage is applied at the node of the mode shapes, such as DMG2 at the 2nd and 4th bending modes, the mode shapes are little affected.

- Torsion modes become more dominant as damage is applied either asymmetrically or on an element against large design dead load, such as the torsion mode in DMG3 scenario. In some cases, torsion modes become more dominant in a modemixture manner, i.e. in a mode mixed with a certain bending mode, but not alone, such as the mode mixed with the 3rd bending mode in DMG3 scenario.
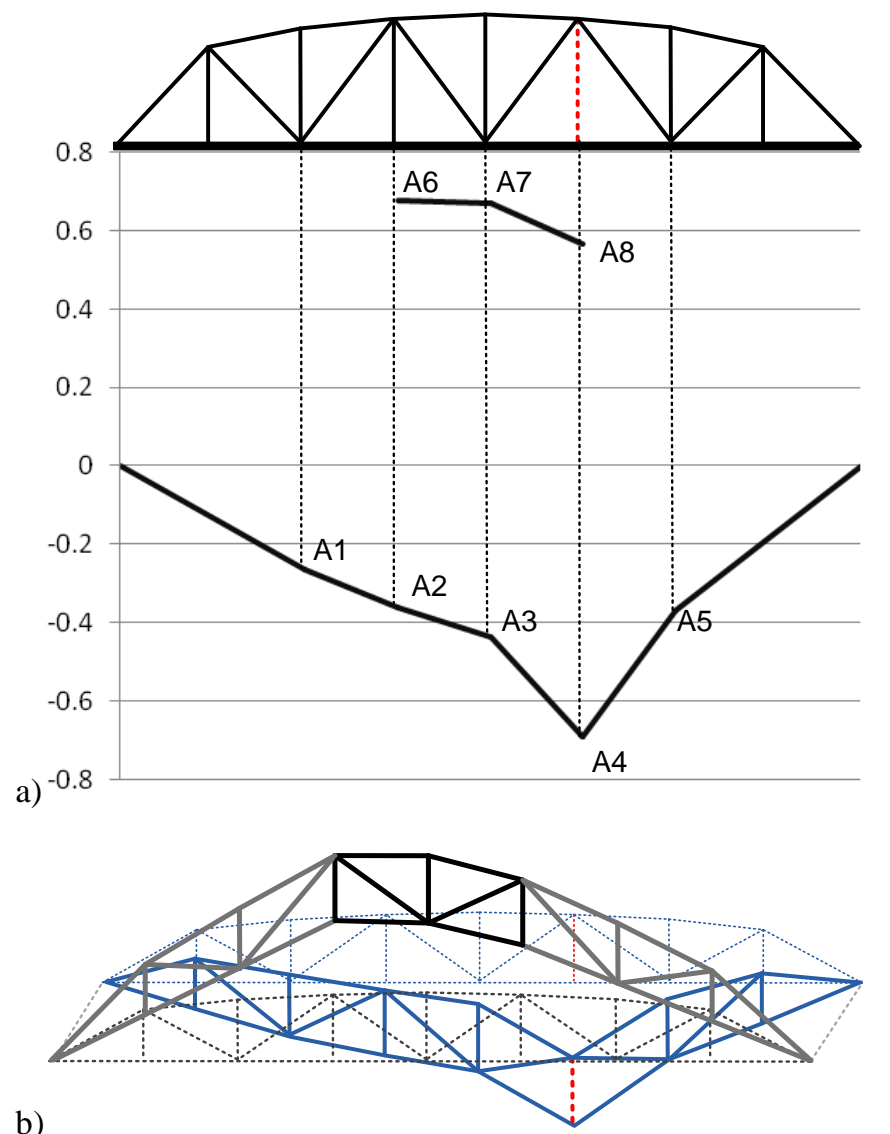

Figure 8. Mean mode shape of the first torsion mode: a) identified for DMG3 scenario; b) 3-D behavior.

\section{CONCLUDING REMARKS}

From the field damage experiments conducted on the real simply-supported steel truss bridge, several concluding remarks can be drawn from statistical properties of modal parameters as follows.

- Mean values of the modal frequencies suggested to interpret the identified modal parameters of lower modes from bridge free-vibration responses, for higher resolution and precision purposes, and of higher modes that are available exclusively from forced-vibration responses. 
- However, the coefficient of variance of the modal frequencies showed that free vibration data offer more precise results than forced vibrations do.

- Mean values of the modal parameters are little affected by damage causing low stress redistribution.

- Mean mode shapes showed that modal frequencies decrease, signifying a global stiffness loss, as damage causing high stress redistribution is applied. Such a change can be observed if the damage is applied at the non-nodal point of corresponding mode shapes, but it is not necessarily observable if the damage is at the node.

- Mean mode shapes of both symmetric and antisymmetric mode are distorted as asymmetric damage is applied but are not as symmetric damage is applied. Alternatively, as damage is applied at the non-nodal point of mode shapes, the mode shapes show an amplification in the damaged side (equivalent to a reduction in the opposite side), reflecting a stiffness reduction in this side; as damage is applied at the node of the mode shapes, the mode shapes are little affected.

- Torsion modes become more dominant as damage is applied either asymmetrically or on an element against large design dead load.

The above concluding remarks drawn herein strictly apply to bridge types and damage scenarios similar to those in this experimental study only. More general applicability requires more field tests on various real bridges and damage scenarios.

\section{ACKNOWLEDGEMENT}

This study is partly sponsored by Japanese Society for the Promotion of Science (JSPS) for the Grantin-Aid for Scientific Research (B) under project No. 24360178. The second author, K.C. Chang, is sponsored by "The JSPS Postdoctoral Fellowship for Foreign Researchers" Program. Such financial aids are gratefully acknowledged. The authors wish to express their gratitude to Nara Prefecture; to Dr. Oshima, Mr. Lee, and Ms. Nakamura of Kyoto University; and to Mr. Kaneda of Osaka City University for carrying out the field experiments.

\section{REFERENCES}

Brincker, R., Zhang, L., \& Andersen, P. 2000. Modal identification from ambient responses using frequency domain decomposition, Proceedings of the 18th International Modal Analysis Conference: 625-630.

Brincker, R., Zhang, L., \& Andersen, P. 2001. Modal identification of output-only systems using frequency domain decomposition, Smart Materials and Structures 10: 441-445.

Carden E.P. \& Fanning, P. 2004. Vibration based condition monitoring: A review, Structural Health Monitoring 3(4): 355-377.

Chang, K.C., Kim, C.W. \& Borjigin, S. 2013. Variability in bridge frequency induced by a parked vehicle, Smart Structures and Systems, to appear.

Dilena, M. \& Morassi, A. 2011. Dynamic testing of a damaged bridge, Mechanical Systems and Signal Processing 25: 1485-1507.

Fan, W. \& Qiao, P. 2011. Vibration-based damage identification methods: a review and comparative study, Structural Health Monitoring 10(1): 83-111.

Farrar, C.R., Baker, W.E., Bell, T.M., Cone, K.M., Darling, T.W., Duffey, T.A., Eklund, A. \& Migliori, A. 1994. Dynamic characterization and damage detection in the I-40 Bridge over the Rio Grande, Los Alomos National Laboratory report: LA-12767-MS.

Farrar, C.R., Doebling, S.W. \& Nix, D.A. 2001. Vibrationbased structural damage identification, Philosophical Transactions of the Royal Society A 359: 131-149.

Furukawa, A. \& Otsuka, H. 2008. Stiffness reduction identification of steel truss bridge using micro shaking device, Proceedings of the 14th World Conference on Earthquake Engineering, No.14-0051.

Huth, O., Feltrin, G., Maeck, J., Kilic, N. \& Motavalli, M. 2005. Damage identification using modal data: experiences on a prestressed concrete bridge, Journal of Structural Engineering, ASCE, 131: 1898-1910.

Kim, C.W., Kitauchi, S. \& Sugiura, K. 2013. Damage detection of a steel bridge through on-site moving vehicle experiments, Proceedings of the Second Conference on Smart Monitoring, Assessment and Rehabilitation of Civil Structures (SMAR2013), Istanbul, Turkey, Sept. 9-11, 2013, to appear.

Kramer, C., De Smet, C.A.M. \& De Roeck, G. 1999. Z24 Bridge damage detection tests, Proceedings of the 17th International Modal Analysis Conference: 1023-1029.

Maas, S., Zurbes, A., Waldmann, D., Waltering, M., Bungard, V. \& De Roeck, G. 2012. Damage assessment of concrete structures through dynamic testing methods. Part 2: Bridge tests, Engineering Structures 34: 483-494.

Yoshioka, T., Takahashi, M., Yamaguchi, H. \& Matsumoto, Y. 2011. Damage assessment of truss diagonal members based on frequency changes in local higher modes, Procedia Engineering 14: 3119-3126. 